\title{
ABO-incompatible liver transplantation using only rituximab for patients with low anti-ABO antibody titer
}

\author{
Boram Lee ${ }^{1}$, YoungRok Choi ${ }^{1}$, Ho-Seong Han ${ }^{1}$, Yoo-Seok Yoon ${ }^{1}$, Jai Young Cho', \\ Sook-Hyang Jeong ${ }^{2}$, Jin-Wook Kim², Eun Sun Jang ${ }^{2}$, and Soomin $\mathrm{Ahn}^{3}$ \\ Departments of ${ }^{1}$ Surgery, ${ }^{2}$ Medicine, and ${ }^{3}$ Pathology, Seoul National University Bundang Hospital, \\ Seoul National University College of Medicine, Seongnam, Korea
}

\begin{abstract}
Backgrounds/Aims: Graft survival after ABO-incompatible (ABOi) living donor liver transplantation (LDLT) has increased due to advances in desensitization methods. We analyzed early outcomes following ABOi LDLT using only rituximab without any additional desensitization methods in recipients with low anti-ABO antibody titers ( $\leq 1: 32)$. Methods: Ten adult patients underwent ABOi LDLT between September 2014 and December 2016. All patients were administered a single dose of rituximab $\left(300 \mathrm{mg} / \mathrm{m}^{2}\right)$ prior to LDLT. Three patients with baseline anti-ABO titer $>1: 32$ underwent multiple sessions of plasmapheresis to reduce titers to $<1: 32$ (rituximab+plasmapheresis, RP). Seven patients with low anti-ABO titer $(\leq 1: 32)$ did not undergo plasmapheresis (rituximab-only, RO). ABO-compatible LDLT patients during the same period were included for comparison $(n=22)$. Results: Post-transplantation titers were significantly lower in the RO than in the RP and showed no rebound rise (POD7 1.14 \pm 0.38 vs 28.0 $\pm 31.7, p=0.04$ ), (POD30 1.26 \pm 0.45 vs $108 \pm 107, p=0.02)$. There were no significant differences in rejection, biliary complications and infection between groups. There were no significant differences in outcome between the RO group and ABO-compatible except for infection. Conclusions: This study shows that recipients with low baseline anti-ABO antibody titer $(\leq 1: 32)$ can undergo $A B O i$ LDLT using conventional immunosuppression and rituximab alone. (Ann Hepatobiliary Pancreat Surg 2019;23:211-218)
\end{abstract}

Key Words: Antibody mediated rejection; Anti-ABO antibody titer; Plasmapheresis; Rituximab; Liver transplant

\section{INTRODUCTION}

ABO-incompatible (ABOi) living donor liver transplantation (LDLT) is an attractive option for expanding donor pools. Although early experiences were disappointing, recipient outcomes have improved because of the advances in desensitization protocols. Song ${ }^{1}$ reported 3-year graft and patient survival rates are following ABOi LDLT of $86.5 \%$ and $87.6 \%$, respectively, comparable to those following ABO-compatible (ABOc) LDLT. The main aims of desensitization protocols are to eliminate preformed anti-ABO antibodies, to deplete serum B cells, and to reduce immune reactions. However, there is no standardized desensitization protocol for ABOi LDLT.

Plasmapheresis (PP) plays an important role in ABOi LDLT by lowering anti-ABO antibody titer before and af- ter transplantation. Egawa et al. $^{2}$ have reported that all long-term survivors showed lower levels of anti-ABO antibody titers, while Ashizawa et al. ${ }^{3}$ reported that elevation of anti-ABO antibody titers after transplantation may be a predictive risk factor for increased transplantationrelated mortality and morbidity. Plasmapheresis is an indispensable treatment for improving the outcomes of ABOi LT.

However, plasmapheresis for liver transplantation is classified as category $\mathrm{III}^{4}$ according to the American Society for Apheresis guidelines (Category III: optimum role of apheresis therapy is not established; decision-making should be individualized). Because the procedure requires a largebore catheter and is necessarily invasive, some complications inevitably arise. The complication rate of plasmapheresis in LDLT patients is estimated at $25-40 \%{ }^{5,6}$ Song

Received: November 22, 2018; Revised: March 17, 2019; Accepted: March 29, 2019

Corresponding author: YoungRok Choi

Department of Surgery, Seoul National University Bundang Hospital, Seoul National University College of Medicine, 82 Gumi-ro 173 Beon-gil, Bundang-gu, Seongnam 13620, Korea

Tel: +82-31-787-7111, Fax: +82-31-787-4055, E-mail: choiyoungrok@gmail.com

Copyright (C) 2019 by The Korean Association of Hepato-Biliary-Pancreatic Surgery

This is an Open Access article distributed under the terms of the Creative Commons Attribution Non-Commercial License (http://creativecommons.org/ licenses/by-nc/4.0) which permits unrestricted non-commercial use, distribution, and reproduction in any medium, provided the original work is properly cited. Annals of Hepato-Biliary-Pancreatic Surgery - pISSN: 2508-5778 - elSSN: 2508-5859 
et al. have reported that initial and post-LT peak levels of anti-ABO antibody titers were not associated with the incidence of antibody mediated rejection (AMR) in univariate analysis. ${ }^{7}$ Furthermore, Egawa et al. ${ }^{8}$ have concluded that only the absence of rituximab was a significantly risk factor for AMR in a multivariate analysis. Therefore, it is doubtful that plasmapheresis is necessary for all patients in ABOi LDLT and may be contraindicated due to the high risk of complication. This study aims to analyze early outcomes after ABOi LDLT using only rituximab without plasmapheresis in recipients with low anti-ABO antibody titer $(\leq 1: 32)$.

\section{PATIENTS AND METHODS}

\section{Patients}

Ten patients underwent adult ABOi LDLT (age $>18$ years) and twenty-two patients underwent adult ABOcompatible (ABOc) LDLT between September 2014 and December 2016 at Seoul National University Bundang Hospital. Among the ABOi LDLT group, seven patients had a low titer of anti-ABO antibody $(\leq 1: 32)$ at the time of admission.

All recipients received a modified right liver from a living donor. All donors were healthy people without underlying disease. Transplanted livers had microvesicular fatty change of less than $10 \%$. The medical records of all patients were retrospectively reviewed. All operation were performed by the same surgical team.

\section{Desensitization protocol and post-LT immuno- suppression}

The same triple immunosuppression protocol was used for both $\mathrm{ABOc}$ and $\mathrm{ABOi}$ LDLT recipients; the regimens differed in that $\mathrm{ABOc}$ patients received basiliximab as induction therapy and $\mathrm{ABOi}$ patients received rituximab as desensitization therapy. The triple regimen consisted of tacrolimus (target level: $8-12 \mathrm{ng} / \mathrm{ml}$ for 6 months, $6-8 \mathrm{ng} / \mathrm{ml}$ between 6 and 12 months post-LT), mycophenolate mofetil (500 mg twice per day) and steroids (methylprednisolone, $1000 \mathrm{mg}$ tapering to $20 \mathrm{mg}$ /day). Splenectomy and local infusion therapy were not performed routinely. When performing the ABOc LDLT, we administered basiliximab (Simulect, Novartis, Montreal, Quebec, Canada) as an induction therapy, $20 \mathrm{mg}$ on the day of operation and the same on post-operative day 4. In case of ABOi LDLT, we did not inject the basiliximab. All patients who underwent ABOi LDLTs were administered a single dose of rituximab (RTX) (Rituxan \& MabThera, Roche, Switzerland) (300 mg/m $\mathrm{m}^{2}$ of body surface area) 1-3 weeks before LDLT. Three ABOi patients with high baseline anti-ABO titer $(>1: 32)$ underwent multiple sessions of plasmapheresis to reduce the preformed anti-ABO antibody titers to $\leq 1: 32$ or lower (rituximab+plasmapheresis, RP). Patients with baseline anti-ABO titer below the $\leq 1: 32$ did not undergo plasmapheresis (rituximab-only, RO) (Fig. 1). We use intravenous immunoglobulin (IVIg) for patients with high $\mathrm{ABO}$ antibody titer, high panel reactive antibody (PRA) and strong cross matching results.

\section{Infection prophylaxis}

Perioperative protocols for the prevention of infection were the same in both ABOi and ABOc LDLT groups: patients received prophylactic antibiotics: $3 \mathrm{~g}$ ampicillin sulbactam intravenously every 6 hours, until postoperative day 2; prophylactic antifungal: $100 \mathrm{mg}$ of fluconazole during the first four postoperative weeks, sulfamethoxazole and trimethoprim (Bactrim, Roche, Switzerland) $480 \mathrm{mg}$ twice a week, for the first postoperative year; and anti-cytomegalovirus (CMV) for high risk patients (CMV Immunoglobulin $\mathrm{G}(+)$ donor to CMV Immunoglobulin $\mathrm{G}(-)$ recipient).

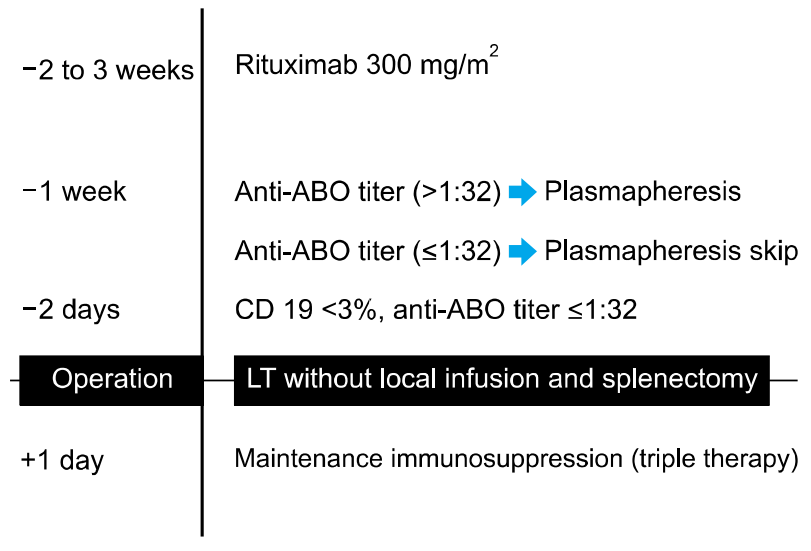

Fig. 1. Protocol for ABO-incompatible living donor liver transplantation. LT, liver transplantation; SNUBH, Seoul National University Bundang Hospital. 


\section{Monitoring for Anti-ABO antibody titer, CD19+ lymphocyte count and rejection}

Basal levels of the pre-formed anti-ABO antibody titer and CD19+ lymphocyte count were checked before the administration of RTX and serially measured after the initiation of plasmapheresis. Our center used the serial test tube dilution method for determination of the liver transplantation recipient's isoagglutinin titer. After the ABOi LDLT, the anti-ABO antibody titer was checked on postoperative day $7,14,21,28$. To confirm the effect of RTX, we checked serum CD19+ lymphocyte count before LDLT and the count was rechecked at post-operative day 7 .

To identify rejection after transplantation, we performed routine protocol liver biopsy and CT scan at postoperative day 7 .

\section{Rejection and complications}

Transplantation-related complications included biliary complication, infectious complication and incidence of acute antibody mediated rejection (AMR), acute cellular rejection (ACR) were measured. Biliary complications were considered meaningful when surgical, endoscopic, or radiologic interventions were required. ( $\geq$ IIIa, Clavin-Dindo classification).

ACR biopsy specimens were graded using Banff schema, the international standard for grading acute liver allograft rejection. This schema is composed of two parts, the global assessment (GA) and the rejection activity index (RAI). ${ }^{9}$ We determined the presence of acute AMR histopathologically, using the Banff criteria (including the following: portal microvascular endothelial cell hypertrophy, portal capillary and inlet venule dilatation, monocytic, eosinophilic, and neutrophilic portal microvasculitis, portal edema, ductular reaction; cholestasis usually present, but variable; edema and periportal hepatocyte necrosis, which are more common/prominent in ABOi allograft; variable active lymphocytic and/or necrotizing arteritis); positive serum donor-specific antibody (DSA); diffuse microvascular $\mathrm{C} 4 \mathrm{~d}$ deposition on frozen or formalin-fixed, paraffin-embedded tissue in liver allograft; and reasonable exclusion of other insults that might cause a similar pattern of injury. ${ }^{10}$

\section{Statistical analysis}

All statistical analyses were performed by using IBM SPSS Statistics ver. 18.0 (IBM Co., Armonk, NY, USA). Patient demographics are expressed as median (range). The Mann-Whitney $U$ test was used for comparisons between the ABOi and ABOc LDLTs.

\section{RESULTS}

\section{Patient characteristics}

i) ABOi LDLT (rituximab-only, RO) versus ABOi LDLT (rituximab+plasmapheresis, RP)

A total of 10 ABOi LDLTs were performed. Seven patients with low titer $(\leq 1: 32)$ underwent ABOi LDLT receiving standard triple immunosuppression without plasmapheresis ( $\mathrm{RO}$ group, $\mathrm{n}=7$ ). The remaining three patients underwent standard triple immunosuppression and multiple sessions of plasmapheresis until the titer fell below $\leq 1: 32$ (RP group, $n=3$ ). General characteristics of ABOi $\mathrm{LT}$ recipients are shown in Table 1 . There were no significant differences between the RO group and RP group. The RO group showed slightly higher mean pre-transplant Model for End-stage Liver Disease (MELD) scores than RP group $(17.23 \pm 7.61$ vs $9.67 \pm 3.79, p=0.31)$, but there was no significant difference. The time interval between administration of RTX and LT average length of time that properties was 6.71 (3-13) days in the RO group and 17.3 (13-20) days in the RP group. Detailed clinical information about ABOi LDLT patients is shown in Table 2. Although two patients (Patient $4=20 \%$, Patient $7=15 \%$ ) showed a relatively high level of baseline serum CD19+ lymphocytes, it was effectively suppressed after administration of a single dose of RTX before transplantation. Patients belonging to the RP group received an average of three cycles of plasmapheresis.

ii) ABOi LDLT (rituximab-only, RO) versus ABOc LDLT There was no significant difference in the general characteristics of the RTX-only ABOi group and the ABOc group (Table 3).

\footnotetext{
Changes in the $C D 19+B-$ cell ratio and anti-ABO antibody titers in the $A B O i$ group

i) Change in CD19+ counts

There was no significant difference in initial (baseline
} 
Table 1. Comparison of demographics between the rituximab-only (RO) and rituximab+plasmapheresis (RP) groups in ABOi Adult LDLT

\begin{tabular}{lccc}
\hline \multicolumn{1}{c}{ Variable } & RO group $(\mathrm{n}=7)$ & RP group $(\mathrm{n}=3)$ & $p$-value \\
\hline Recipient age & $51.7(40-61)$ & $58.7(53-66)$ & 0.21 \\
Sex (Male/Female) & $3 / 4$ & $3 / 0$ & 0.20 \\
ABO blood type & 0 & $3(100 \%)$ & 0.06 \\
O+ & $4(57.1 \%)$ & 0 & 0.48 \\
A+ & $3(42.9 \%)$ & 0 & \\
B+ & 0 & $3(100 \%)$ & \\
AB+ & $4(57.1 \%)$ & 0 & 0.47 \\
Original disease & $1(14.3 \%)$ & 0 & \\
HBV & $2(28.6 \%)$ & & \\
HCV & & & \\
alcohol & $4(57.1 \%)$ & $3(100 \%)$ & 0.31 \\
Autoimmue & $3(42.9 \%)$ & $0.67(7-14)$ & 0.47 \\
Combined HCC & $17.23(8-28)$ & $0.82(0.77-0.9)$ & 0.26 \\
Yes & $1.08(0.86-1.38)$ & $17.3(13-20)$ & \\
No & $6.71(3-13)$ & & \\
MELD score (range) & & \\
GRWR(\%) & & & \\
RTX-LT intervals (day) & & & \\
\hline
\end{tabular}

RTX, rituximab; PP, plasmapheresis; HBV, hepatitis B virus; HCV, hepatitis C virus; HCC, hepatocellular carcinoma; MELD, Model for End-Stage Liver Disease; GRWR, graft-to-recipient body weight ratio; RTX-LT intervals, the time between administration of RTX and liver transplantation

Table 2. Clinical information of ABOi adult LDLT group

\begin{tabular}{|c|c|c|c|c|c|c|c|c|}
\hline $\begin{array}{l}\text { Case } \\
\text { No }\end{array}$ & $\begin{array}{c}\text { Sex/ } \\
\text { age }\end{array}$ & Etiology & MELD & $\begin{array}{l}\text { Blood type } \\
\text { (R/D) }\end{array}$ & $\begin{array}{c}\text { Initial } \\
\text { CD19+ } \\
\text { counts }(\%)\end{array}$ & $\begin{array}{c}\text { Initial anti-ABO } \\
\text { Ab titer (IgM / IgG) }\end{array}$ & $\begin{array}{l}\text { RTX injection } \\
\text { - LT interval } \\
\text { (days) }\end{array}$ & $\begin{array}{l}\text { Pre-LT } \\
\text { PP }\end{array}$ \\
\hline 1 & $\mathrm{~F} / 50$ & $\mathrm{AIH}$ & 27 & $\mathrm{~B}+/ \mathrm{AB}+$ & $4 \%$ & $1: 8 / 1: 8$ & 3 & $x$ \\
\hline 2 & $\mathrm{M} / 51$ & $\begin{array}{l}\text { HBV-LC combined } \\
\text { CCC-HCC }\end{array}$ & 13 & $\mathrm{~B}+/ \mathrm{AB}+$ & $5 \%$ & $<1: 2 /<1: 2$ & 8 & $x$ \\
\hline 3 & $\mathrm{M} / 61$ & $\mathrm{HCC}$ & 8 & $\mathrm{~A}+/ \mathrm{B}+$ & $10 \%$ & $1: 32 / 1: 32$ & 4 & $x$ \\
\hline 4 & $\mathrm{~F} / 51$ & AIH & 28 & $\mathrm{~B}+/ \mathrm{AB}+$ & $20 \%$ & $1: 8 / 1: 8$ & 3 & $x$ \\
\hline 5 & $\mathrm{~F} / 48$ & Alcoholic LC & 15 & $\mathrm{~A}+/ \mathrm{AB}+$ & $3 \%$ & $1: 4 / 1: 4$ & 7 & $x$ \\
\hline 6 & $\mathrm{~F} / 48$ & HBV LC HCC & 18 & $\mathrm{~A}+/ \mathrm{B}+$ & $1 \%$ & $1: 8 / 1: 8$ & 7 & $x$ \\
\hline 7 & $60 / \mathrm{M}$ & HCC LC & 12 & $\mathrm{~A}+/ \mathrm{B}+$ & $15 \%$ & $1: 16 / 1: 16$ & 13 & $\times$ \\
\hline 8 & $\mathrm{M} / 57$ & HBV LC HCC & 7 & $\mathrm{O}+/ \mathrm{A}+$ & $4 \%$ & $1: 32 / 1: 2$ & 20 & 3 cycles \\
\hline 9 & $\mathrm{M} / 53$ & HBV LC HCC & 8 & $\mathrm{O}+/ \mathrm{AB}+$ & $11 \%$ & $\begin{array}{ll}\text { A: } 1: 32 / 1: 2 \\
\text { B: } 1: 32 / 1: 2\end{array}$ & 19 & 4 cycles \\
\hline 10 & $\mathrm{M} / 66$ & HBV LC HCC & 14 & $\mathrm{O}+/ \mathrm{A}+$ & $16 \%$ & $1: 16 / 1: 8$ & 13 & 2 cycles \\
\hline
\end{tabular}

$\mathrm{R} / \mathrm{D}$, recipient blood type/donor blood type; Ab, antibody; PP, plasmapheresis; AIH, autoimmune hepatitis; CCC, cholangiocarcinoma; LC, liver cirrhosis; HCC, hepatocellular carcinoma; RTX-LT intervals, the time between administration of RTX and liver transplantation; LT, liver transplantation

counts before administration of RTX), pre-transplantation or 1-week-post-transplantation serum CD19+ lymphocyte ratio between RO group and RP group (Fig. 2). Although the serum CD19+ lymphocyte count at 1 week after transplantation was higher in the RO group, there was no significant difference (RO group, 3.71 \pm 4.50 ; RP group, $0.67 \pm$ $0.58 ; p=0.56)$. ii) Changes in anti-ABO antibody titers

The changes in the anti-ABO antibody titers in ABOi LDLT are shown in Fig. 3. The average initial anti-ABO antibody titer was $20.0 \pm 21.5$ in the RO group and $213 \pm$ 73.9 in RP group. Because plasmapheresis was not performed in the low anti-ABO antibody titer group $(\leq 1: 32)$, the initial anti-ABO antibody titer was significantly lower 
Table 3. Comparison of demographics between $\mathrm{ABOc}$ and rituximab-only (RO) group ABOi Adult LDLT

\begin{tabular}{|c|c|c|c|}
\hline Variable & ABOi group (RO group) $(n=7)$ & ABOc group $(n=22)$ & $p$-value \\
\hline Recipient age & $51.7(40-61)$ & $52.6(35-76)$ & 0.45 \\
\hline $\operatorname{Sex}(M / F)$ & $3 / 4$ & $15 / 7$ & 0.46 \\
\hline ABO blood type & & & 0.33 \\
\hline $\mathrm{O}+$ & 0 & $4(18.2 \%)$ & \\
\hline $\mathrm{A}+$ & $4(57.1 \%)$ & $8(36.4 \%)$ & \\
\hline $\mathrm{B}+$ & $3(42.9 \%)$ & $7(31.8 \%)$ & \\
\hline $\mathrm{AB}+$ & 0 & $3(13.6 \%)$ & \\
\hline Original disease & & & 0.38 \\
\hline HBV & $4(57.1 \%)$ & $12(54.5 \%)$ & \\
\hline $\mathrm{HCV}$ & 0 & $1(4.55 \%)$ & \\
\hline Alcohol & $1(14.3 \%)$ & 0 & \\
\hline Autoimmue & $2(28.6 \%)$ & 0 & \\
\hline Combined HCC & & & 0.49 \\
\hline Yes & $4(57.1 \%)$ & $9(64.3 \%)$ & \\
\hline No & $3(42.9 \%)$ & $5(35.7 \%)$ & \\
\hline Emergency LT & & & 0.28 \\
\hline Yes & 0 & $2(9.1 \%)$ & \\
\hline No & $7(100 \%)$ & $20(90.9 \%)$ & \\
\hline MELD (score) & $17.23(8-28)$ & $20.2(6-38)$ & 0.34 \\
\hline Donor age & $31.4(19-45)$ & $33.1(19-49)$ & 0.22 \\
\hline GRWR (\%) & $1.08(0.86-1.38)$ & $1.09(0.7-1.56)$ & 0.56 \\
\hline
\end{tabular}

HBV, hepatitis B virus; HCV, hepatitis C virus; HCC, hepatocellular carcinoma; LT, liver transplantation; MELD, Model for End-Stage Liver Disease; GRWR, graft-to-recipient body weight ratio

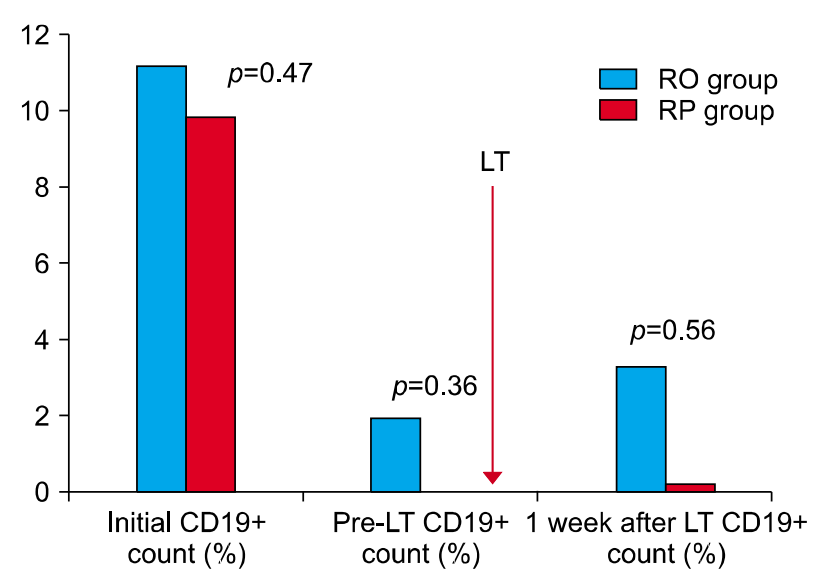

Fig. 2. Changes in the CD19+ lymphocyte count in ABOi adult LDLT. ABOi, ABO-incompatible; LDLT, living donor liver transplantation; RO, Rituximab only; RP, Rituximab+ Plasmapheresis; LT, Liver transplantation.

in the RO group ( $p=0.03)$. The anti-ABO antibody titer before the transplantation was reduced to less than the target of 1:32 in both groups. (RO group, 9.29 \pm 10.4 ; RP group, 26.67 $\pm 9.24 ; p=0.14$ ). When comparing the antiABO antibody titer after a week of transplantation, the titer was kept low in RO group (RO group, $1.14 \pm 0.38$; RP

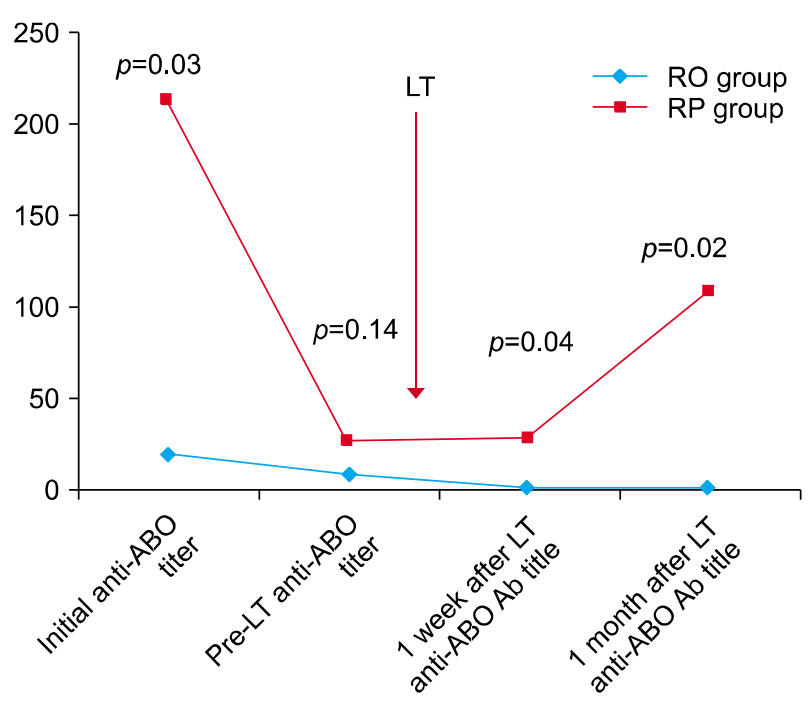

Fig. 3. Changes in the Anti-ABO antibody titer in ABOi adult LDLT. ABOi, ABO-incompatible; LDLT, living donor liver transplantation; RO, Rituximab only; RP, Rituximab+Plasmapheresis; LT, Liver transplantation.

group, 28.0 $\pm 31.7 ; p=0.04$ ). The RO group did not show the post-transplantation rebound rise, and the titer remained low a month after transplantation (RO group, 1.26 \pm 0.45; RP group, $108 \pm 107$; $p=0.02$ ). 
Table 4. Comparison of post-LT morbidities between rituximab-only (RO) and rituximab+plasmapheresis (RP) in ABOi Adult LDLT

\begin{tabular}{lccc}
\hline & RO group $(\mathrm{n}=7)$ & RP group (n=3) & $p$-value \\
\hline ACR & $1(14.3 \%)$ & 0 & 1.00 \\
AMR & 0 & 0 & 1.00 \\
Biliary complication & $2(28.6 \%)$ & 0 & 0.40 \\
$\quad$ Stricture & $2(28.6 \%)$ & $1(33.3 \%)$ & 0.50 \\
$\quad$ leakage & 0 & 0 & 0.71 \\
DIHBS & $4(57.1 \%)$ & $1(33.3 \%)$ & 1.00 \\
Infection & $2(28.6 \%)$ & $1(33.3 \%)$ & \\
Malignancy recurrence & 0 & 0 & 0 \\
Anastomosis complication & $1(14.3 \%)$ & 0 & \\
Hepatic artey stenosis & 0 & 0 & \\
Hepatic vein stenosis & 0 & 0 & \\
Portal vein stenosis & & 0 & \\
30-day mortality & & & \\
\hline
\end{tabular}

ACR, acute cellular rejection; AMR, antibody-mediated rejection; DIHBS, diffuse intrahepatic biliary stenosis

Table 5. Comparison of complications after transplantation between rituximab-only (RO) group ABOi Adult LDLT and ABOc LDLT

\begin{tabular}{lccc}
\hline & ABOi group (RO group) $(\mathrm{n}=7)$ & ABOc group $(\mathrm{n}=22)$ & $p$-value \\
\hline ACR & $1(14.3 \%)$ & $2(9.1 \%)$ & 0.90 \\
AMR & 0 & 0 & 0.22 \\
Biliary complication & $2(28.6 \%)$ & $6(27.3 \%)$ & 0.38 \\
$\quad$ Stricture & $2(28.6 \%)$ & $1(4.5 \%)$ & 0.02 \\
$\quad$ leakage & 0 & 0 & 0.14 \\
$\quad$ DIHBS & $4(57.1 \%)$ & $2(9.1 \%)$ & 1.00 \\
Infection & $2(28.6 \%)$ & $1(4.5 \%)$ & \\
Malignancy recurrence & & 0 & 1.00 \\
Anastomosis complication & 0 & $1(4.5 \%)$ & $1(4.5 \%)$ \\
$\quad$ Hepatic artey stenosis & $1(14.3 \%)$ & $1(4.5 \%)$ & \\
$\quad$ Hepatic vein stenosis & 0 & 0 & \\
$\quad$ Portal vein stenosis & 0 & & \\
30-day mortality & & & \\
\hline
\end{tabular}

ACR, acute cellular rejection; AMR, antibody-mediated rejection; DIHBS, diffuse intrahepatic biliary stenosis

\section{Outcomes and morbidity}

i) ABOi LDLT (rituximab-only, RO) versus ABOi LDLT (rituximab+plasmapheresis, RP)

There were no significant differences in transplantationrelated complications between the RO group and RP group (Table 4). The biliary anastomosis site stricture and biliary anastomosis site leakage rate ( $\geq$ IIIa, Clavin-Dindo classification) of the RO group were $28.6 \%$ and $28.6 \%$, respectively. However, there were no significant differences between the RO group and RP group $(p=0.40)$. All patients were treated with endoscopic retrograde biliary stenting or percutaneous trans-hepatic cholangiographic drainage. There were no cases of diffuse intrahepatic biliary stricture (DIHBS) in either group. There were no significant differ- ences in infection complications between the two groups $(p=0.50)$.

ii) ABOi LDLT (rituximab-only, RO) versus ABOc LDLT

When comparing the early outcomes between RO group ABOi LDLT and ABOc LDLT, there were no significant differences between the two groups except for infection rate. (RO group $\mathrm{ABOi}, 57.1 \%$; $\mathrm{ABOc}, 9.1 \% ; p=0.02$; Table 5). In the RO group, two patients experienced pneumonia and two experienced cholangitis. The 30-day patient and graft survival of the RO group was $100 \%$. However, one patient in the ABOc group died because of primary graft failure on post-operative day 7 . 


\section{Rejection}

No acute AMR occurred in either the ABOi or ABOc LDLT group. In the RO group, one patient developed ACR. The liver needle biopsy on post-operative day 7 showed mild infiltration of lymphocytes and a few neutrophils in most portal tracts $(\mathrm{RAI}=5)$. Rejection therapy was performed because the hepatic enzyme levels were elevated. After the steroid pulse therapy, the histology showed improvement on post-operative day 13. In the case of the ABOc LDLT group, only ACR occurred $(n=2$, $9.1 \%$ ). Both patients were treated with steroid pulse therapy and adjustment of immunosuppressant.

\section{DISCUSSION}

We report our early experiences that recipients with low baseline anti-ABO antibody titers can successfully undergo ABOi LDLT using only rituximab without plasmapheresis. Although ABOi graft survival has been improved with desensitization, AMR is still considered a difficult problem. A previous study reported that the incidence of AMR decreased from $23.5 \%$ to $6.2 \%$ after the introduction of RTX. ${ }^{7}$ In our cases, acute AMR did not occur in either the RTX-only or RTX+PP ABOi LDLT group. Additionally, during the follow-up period, there was no death in the ABOi LDLT group (median follow-up period: 22 months).

Since the pre-formed anti-ABO antibody was demonstrated to mediate hyperacute or severe rejection in $\mathrm{ABO}$ $\mathrm{LDLT}^{9}$, there have been several reports regarding the relationship between anti-ABO antibody titer and acute AMR. Because the presence of anti-ABO antibody in the recipient is a risk for AMR, reducing antibody levels plays a key role in the success of the transplantation. ${ }^{10}$ Another study reported that high preoperative antibody titer did not have a significant effect on AMR, instead emphasizing the importance of preventing new antibody production after transplantation. ${ }^{11}$ Although the amount of acceptable pretransplantation anti $\mathrm{ABO}$ antibody titer is not standardized for ABOi LDLT, desensitization protocols have been directed toward the elimination of anti-ABO antibody titers and suppression of $\mathrm{B}$ cell activity before and after transplantation.

Nevertheless, there were three reasons why we did not perform plasmapheresis in the patients with low baseline anti-ABO antibody. First, plasmapheresis, by definition, requires the removal of a large volume of plasma. The volume removed is such that if it is not replaced, significant hypovolemia resulting in vasomotor collapse would occur. ${ }^{12}$ In our center, the patient with baseline anti-ABO antibody titer (1:128) showed life-threatening hypotension during the plasmapheresis and coagulopathy after the procedure. That is why he did not complete the planned plasmapheresis. Although the patient's titer did not decrease, he underwen ABOi LDLT without any complications or rejection. As mentioned above, the complication rates of plasmapheresis observed in other studies is estimated at 25-40\%. ${ }^{5,6}$ Adverse side effects observed during plasmapheresis included: fall in blood pressure (8.4\%), arrhythmia (3.5\%), and sensation of cold with temporarily elevated temperature and paresthesia (1.1\%). Therefore, advantages and disadvantages must be considered. Second, conventional suppression alone has been shown to be successful in selected kidney transplant recipients with low anti-ABO antibody titers. ${ }^{13}$ Third, according to Yamamoto et al, RTX-only treatment was significantly more cost-effective than the other methods. ${ }^{14}$ Unlike the Yamamoto groups, however, we applied the RO regimen only in low anti-ABO antibody titers.

There was no significant difference in LT-related complications between the RO group and RP group. In addition, the RO group showed low anti-ABO antibody titer after transplantation and did not show a rebound rise of antibody titer after transplantation. AMR has been observed in ABOi KT patients with a high baseline titer. ${ }^{15}$ Despite conflicting views regarding the importance of baseline anti-ABO antibody titers in ABOi LT, our finding that post-transplant antibody levels remained low in patients with low baseline levels indicate that baseline anti-ABO antibody titer is likely to be more important than preoperative titer in determining outcomes.

RTX has increased the graft survival following ABOi. Despite its clear utility, it may have disadvantages as well. The increased incidence of infection we observed in the $\mathrm{RO} A B O i$ group relative to the $\mathrm{ABOc}$ group was also reported in another study. ${ }^{8}$ However, this differs from the results of Egawa et al., who reported no significant differences between the incidence of bacterial infection or CMV disease between RTX and non-RTX groups. ${ }^{16}$ Fur- 
ther research is warranted to investigate the relationship of RTX to infection rates.

This study has several limitations. First, our necessarily small sample size limited robustness, Further studies including larger numbers of patients are needed Second, because the follow-up period of the current study was relatively short, results of a long-term follow-up are needed. Third, since the standard anti-ABO antibody target titer before transplantation has not been established yet, the criteria for low titer $(\leq 1: 32)$ have been set by our center.

In conclusion, LDLT recipients with low baseline anti-ABO antibody titer $(\leq 1: 32)$ can successfully undergo ABOi LDLT using conventional immunosuppression and rituximab alone.

\section{REFERENCES}

1. Song GW. ABO incompatability in liver transplantation. Hanyang Med Rev 2014;34:202-210.

2. Egawa H, Oike F, Buhler L, Shapiro AM, Minamiguchi S, Haga $\mathrm{H}$, et al. Impact of recipient age on outcome of ABO-incompatible living-donor liver transplantation. Transplantation 2004;77: 403-411.

3. Ashizawa T, Matsuno N, Yokoyama T, Kihara Y, Kuzuoka K, Taira S, et al. The role of plasmapheresis therapy for perioperative management in ABO-incompatible adult living donor liver transplantation. Transplant Proc 2006;38:3629-3632.

4. Winters JL. Plasma exchange: concepts, mechanisms, and an overview of the American Society for Apheresis guidelines. Hematology Am Soc Hematol Educ Program 2012;2012:7-12.

5. Mokrzycki MH, Kaplan AA. Therapeutic plasma exchange: complications and management. Am J Kidney Dis 1994;23:817-827.

6. Passalacqua S, Staffolani E, Busnach G, Roccatello D, Pasquali S, Cappelli P, et al.; Apheresis Study Group of the Italian
Society of Nephrology. The Italian Registry for therapeutic apheresis. A report from the Apheresis Study Group of the Italian Society of Nephrology. J Clin Apher 2005;20:101-106.

7. Song GW, Lee SG, Hwang S, Kim KH, Ahn CS, Moon DB, et al. ABO-incompatible adult living donor liver transplantation under the desensitization protocol with rituximab. Am J Transplant 2016;16:157-170.

8. Egawa H, Teramukai S, Haga H, Tanabe M, Mori A, Ikegami $\mathrm{T}$, et al. Impact of rituximab desensitization on blood-type-incompatible adult living donor liver transplantation: a Japanese multicenter study. Am J Transplant 2014;14:102-114.

9. Rummler S, Bauschke A, Baerthel E, Juette H, Maier K, Malessa $\mathrm{C}$, et al. ABO-incompatible living donor liver transplantation in focus of antibody rebound. Transfus Med Hemother 2017;44:4651.

10. Kawagishi N, Satomi S. Current aspects of ABO-incompatible liver transplantation. Clin Case Rep Rev 2016;2:375-379.

11. Krishnan NS, Zehnder D, Briggs D, Higgins R. Human leukocyte antigen antibody incompatible renal transplantation. Indian J Nephrol 2012;22:409-414.

12. Szczepiorkowski ZM, Winters JL, Bandarenko N, Kim HC, Linenberger ML, Marques MB, et al.; Apheresis Applications Committee of the American Society for Apheresis. Guidelines on the use of therapeutic apheresis in clinical practice--evidencebased approach from the Apheresis Applications Committee of the American Society for Apheresis. J Clin Apher 2010;25:83177.

13. Masterson R, Hughes P, Walker RG, Hogan C, Haeusler M, Robertson $\mathrm{AR}$, et al. ABO incompatible renal transplantation without antibody removal using conventional immunosuppression alone. Am J Transplant 2014;14:2807-2813.

14. Yamamoto H, Uchida K, Kawabata S, Isono K, Miura K, Hayashida $\mathrm{S}$, et al. Feasibility of monotherapy by rituximab without additional desensitization in $\mathrm{ABO}$-incompatible living-donor liver transplantation. Transplantation 2018;102:97-104.

15. Gloor JM, Lager DJ, Moore SB, Pineda AA, Fidler ME, Larson $\mathrm{TS}$, et al. ABO-incompatible kidney transplantation using both A2 and non-A2 living donors. Transplantation 2003;75:971-977.

16. Kamar N, Milioto O, Puissant-Lubrano B, Esposito L, Pierre $\mathrm{MC}$, Mohamed AO, et al. Incidence and predictive factors for infectious disease after rituximab therapy in kidney-transplant patients. Am J Transplant 2010;10:89-98. 